\title{
Corrigendum
}

\section{Corrigendum to "The Analysis of Biomechanical Properties of Proximal Femur after Implant Removal"}

\author{
Jae Hyuk Yang, ${ }^{1}$ Tae Gon Jung, ${ }^{2}$ Arjun Rupanagudi Honnurappa, ${ }^{1}$ Jae Min Cha, ${ }^{3}$ \\ Chang Hwa Ham, ${ }^{1}$ Tae Yoon Kim, ${ }^{4}$ and Seung Woo Suh $\mathbb{D D}^{1}$
}

\author{
${ }^{1}$ Scoliosis Research Institute, Department of Orthopedics, Korea University Medical College, Guro Hospital, Guro 2-dong, Guro-gu, \\ Seoul 152-703, Republic of Korea \\ ${ }^{2}$ Osong Medical Innovation Foundation, Medical Device Development Center, Cheongju 363-951, Republic of Korea \\ ${ }^{3}$ The Division of Biological Sciences at The University of Chicago, 5801 South Ellis Avenue Chicago, IL 60637, USA \\ ${ }^{4} J u n g-H w a$ Girls High School, San 105 Beomeo-4-dong Sunsung-gu, Daegu 706-819, Republic of Korea \\ Correspondence should be addressed to Seung Woo Suh; spine@korea.ac.kr
}

Received 4 June 2020; Accepted 22 June 2020; Published 1 August 2020

Copyright (C) 2020 Jae Hyuk Yang et al. This is an open access article distributed under the Creative Commons Attribution License, which permits unrestricted use, distribution, and reproduction in any medium, provided the original work is properly cited.

In the article titled "The Analysis of Biomechanical Properties of Proximal Femur after Implant Removal," [1] it is incorrectly stated that the study received funding from the Korea Healthcare Technology R\&D project. The authors apologise for this error and have clarified that no specific funding was obtained for this research.

\section{References}

[1] J. H. Yang, T. G. Jung, A. R. Honnurappa et al., "The Analysis of Biomechanical Properties of Proximal Femur after Implant Removal," Applied Bionics and Biomechanics, vol. 2016, Article ID 4987831, 10 pages, 2016. 